\title{
Review of the Effectiveness of Vehicle Activated Signs
}

\author{
Diala Jomaa, Siril Yella, Mark Dougherty \\ Department of Computer Engineering, Dalarna University, Borlänge, Sweden \\ Email: djo@du.se,sye@du.se,mdo@du.se
}

Received December 7, 2012; revised January 8, 2013; accepted January 14, 2013

Copyright (c) 2013 Diala Jomaa et al. This is an open access article distributed under the Creative Commons Attribution License, which permits unrestricted use, distribution, and reproduction in any medium, provided the original work is properly cited.

\begin{abstract}
This paper reviews the effectiveness of vehicle activated signs. Vehicle activated signs are being reportedly used in recent years to display dynamic information to road users on an individual basis in order to give a warning or inform about a specific event. Vehicle activated signs are triggered individually by vehicles when a certain criteria is met. An example of such criteria is to trigger a speed limit sign when the driver exceeds a pre-set threshold speed. The preset threshold is usually set to a constant value which is often equal, or relative, to the speed limit on a particular road segment. This review examines in detail the basis for the configuration of the existing sign types in previous studies and explores the relation between the configuration of the sign and their impact on driver behavior and sign efficiency. Most of previous studies show that these signs have significant impact on driver behavior, traffic safety and traffic efficiency. In most cases the signs deployed have yielded reductions in mean speeds, in speed variation and in longer head-ways. However most experiments reported within the area were performed with the signs set to a certain static configuration within applicable conditions. Since some of the aforementioned factors are dynamic in nature, it is felt that the configurations of these signs were thus not carefully considered by previous researchers and there is no clear statement in the previous studies describing the relationship between the trigger value and its consequences under different conditions. Bearing in mind that different designs of vehicle activated signs can give a different impact under certain conditions of road, traffic and weather conditions the current work suggests that variable speed thresholds should be considered instead.
\end{abstract}

Keywords: Vehicle Activated Signs; Variable Message Signs; Threshold Trigger Value; Speed Variation; Traffic Volume; Mean Speed

\section{Introduction}

Excessive or inappropriate speeds are often a reason for traffic fatalities. Therefore the primary consideration for relevant traffic authorities is to reduce speeding either by setting up additional signage or by improving roadways infrastructure. Improving road infrastructure however is quite expensive and hence may not be viable all the time. Therefore, vehicle activated signs (VASs) are recently being used in roadways to increase traffic safety and road efficiency. A VAS is a digital road sign that is mainly used for speed enforcement or to provide the road users with a warning about a hazard. Typically, VAS consists of radar that is mounted inside the sign to detect vehicle or driver speed. The sign displays a message when vehicle speed exceeds a pre-set threshold. It should be noted that VASs belong to a much bigger class of signs known as variable message signs (VMSs). Hence in the current work the terms VAS and VMS have been used interchangeably for the sake of simplicity.
A VMS is a digital board that displays one of a number of messages that may be changed as required in order to inform or warn travelers for a specific event. The message either indicates road and traffic conditions, alternates routes, construction activities or states the appropriate road speed limit. The signs are in general linked to a control center through one to one communication, a local network or radio link to provide real time information on the oncoming road. In the control center, a variety of traffic monitoring and surveillance systems shall extensively be done to provide the right information in order to be displayed to motorists. According to other researcher's definitions, VMS was also known by various other names such as:

- Dynamic Message Sign, DMS;

- Changeable Message Sign, CMS;

- Electronic Message Sign;

- Variable Speed Limit Sign, VSL.

It should be noted that information displayed by a VAS is triggered on an individual basis and provides 
information targeted to a specific individual of the driver population as opposed to VMS which mainly provide information to drivers in general.

Researches reporting the usage of VAS and the more general VMS have both been investigated in this review for the sake of completeness. This review examines in detail the basis for the configuration of the existing sign types in previous studies and explores the relation between the configuration of the sign and their impact on driver behavior and sign efficiency.

Studies reviewing the effect of variable message signs and or vehicle activated signs have been reported by Nygårds and Helmers in 2007 [1]. Such studies have reviewed relevant work published between 2000 and 2005 and have mainly investigated the influence of VMS over human behavior. The authors concluded that VMS have more effect on driver behavior as opposed to static road signs. This is because VMS have higher luminance and better contrast and are hence better at attracting driver's attention. Further the aforementioned study has illustrated that the perceived credibility of the message displayed in the sign plays an essential role in influencing driver behavior. Hence it has been suggested that VMs only display the most relevant information.

More recently another study has reported the happenings within the area during the years 2006-2009 [2]. However, the influence of weather has not been greatly emphasised in the above reported studies i.e. taking into account local weathering conditions prior to displaying information on the VMS. Another major issue that has not been carefully studied concerns the configuration of the sign. Configuration of the signage is deemed extremely important because relevant information collected and displayed by the sign is largely affected as a result of incorrect configuration which in turn greatly affects drivers' compliance. Therefore, once VMS or a VAS is installed, the sign shall not be used as a simple communication device to display various safety messages or unnecessary warning for promoting road safety. The sign must have a solely purpose of providing essential travel advices to drivers. In order to decide whether the traffic advices shall be displayed on or not, most of VMS systems use algorithms based on some threshold values such as traffic flow, occupancy or mean speed or combination of them. These threshold values shall be obviously dynamic and require fine-tuning because different locations in different time can yield different threshold values [3]. Finding a reasonable pre-set threshold value to trigger either VMS or VAS is challenging because an early or late activation of these signs can reduce their eligibility. Besides the reduction of sign eligibility provides to poor compliance between the driver and the established sign.

To apply right configuration on these signs is not a simple task and becomes a key target for transport agen- cies. The threshold should be set depending on road, traffic conditions otherwise the sign face the same problem as in the case of the static signs. Traffic agencies established VMS and VAS in several test sites to evaluate their effect on driver behavior and traffic efficiency but they did not carefully focused on the configuration of the sign in different road conditions. The main research question is how the threshold values were set on these signs and how the signs were configured? At which trigger threshold value should be activated to give positive effect on driver's behavior? Can different threshold values give different impact under adverse conditions? This literature review aims to figure out the following statements:

- The effects of Variable message signs and vehicle activated sigs on driver behavior proving the signs necessity to be related to various weather, road and traffic conditions;

- The parameters used to configure the threshold values that were set on the established signs;

- The parameters used to assess the effectiveness of variable message signs and vehicle activated signs.

The rest of the paper is organised as follows. First, an overview about the assessment of the effectiveness of VMS and VAS has been provided. Next, the effects of variable message sign on driver behavior and headways are provided in Section 3. The effect and evaluation of variable speed limit is presented and discussed in Section 4. Section 5 further discusses the effect of vehicle activated sign. The paper finally presents concluding remarks and provides future research directions.

\section{Assessment of the Effectiveness of VMS and VAS}

There are no universal criteria for measuring the effectiveness of variable message signs. One of the measures is the relationship of the sign to crashes. The collection of crash data correlated to the presence of specific signage is valuable but crashes to a selected test site can be relatively rare. Sometimes may be required several years to determine whether the introduction of the sign had a beneficial effect or not [4]. Therefore the relation between vehicle speed and the frequency of injury collisions had been discussed and interested many researchers. Taylor et al. deducted that for mean speed between 25 $\mathrm{mph}$ to $35 \mathrm{mph}$, the reduction in collisions per $1 \mathrm{mph}$ is $4 \%$ [5]. However, Nilson proposed another relation to estimate the collision reduction based on speed reduction. At $30 \mathrm{mph}$, he concluded that the reduction in speed of 1 mph lead to reduce by $6.6 \%$ in injury accidents, by $0.7 \%$ in serious injury accidents and by $12.7 \%$ in fatal accidents [6]. Another measure that had been used in the effectiveness is changes in drivers' speed. This measure is 
not straightforward in the case for warning signs such as bend curves warning where the speed reduction is not required. The most often measure used in determining warning signs effectiveness is the degree of how much the drivers notice them according to their visibility and comprehensibility. This measurement can be obtained by recall or recognition question to drivers that had been passed recently the signs [4].

\section{Effect of Variable Message Sign on Driver Behaviour and Headways}

A large number of studies had been established to test the effectiveness of VMSs in reducing vehicle speed and in improving safety on a work zone environment. Richard and Alex established a quasi-experiment to examine the effects of different safety messages displayed on VMS on driver's attitudes and on-road traffic speed. The quasiexperiment was chosen on an inter-city Highway 2 between the cities of Edmonton and Calagary. Besides, a questionnaire survey was developed and managed to a sample of 97 drivers to report their responses to the messages displayed on VMS [7]. The results of the survey showed that a small proportion of the respondents reported that the messages increased their likelihood of obeying the speed limit. In addition, there was a small beneficial effect on driver's speed. The safety messages in Richard and Alex study were not reflecting any traffic related information. Their messages were simply a reminder about traffic safety. The messages shown in VMS should only be activated when essential conditions deteriorate otherwise they led to a slightly noticeable effect on driver's speed.

However, Jihzen et al. investigated VMS traffic guide information that was based on real-time detection of traffic flow and the actual conditions in Beijing Olympics. In their study, Jihzen et al. introduced a logical structure of the traffic guidance VMS information system. The system was put into service and performed well during Beijing Olympic Games. The system collected the actual data from the road network, processed it and released timely traffic information to VMS display. The authors claimed to have achieved good practical effects but the assessment of the effectiveness of the system was not shown in this study. The information shown in VMS display was obtained after a complicated storage, processing and computational model. The time taken from the proposed model could lead to late release of the information where it could lead to decreases in VMS reliability.

Firman et al. determined the effectiveness of portable changeable message signs PCMS in rural highway work zones. An experiment was conducted in Seneca, Kansas in US to evaluate PCMS under two different conditions: 1) PCMS was switched on, and 2) PCMS was switched off. The data was collected by two Smart Sensor HD radar sensor systems having the capability of data storage and wireless data downloading. Standard deviation, mean and standard deviation error mean were calculated and analyzed by using statistical software called the Statistical Package for Social Science (SPSS). The results for this experiment showed that when the PCMS was turned on, it reduced vehicle speeds by 4.7 mph over 500 feet distance on average. Besides, when PCMS was turned off, the average speed was reduced to $3.3 \mathrm{mph}$ [8]. Firman et al. did not concerned that there is a reduction in the average speed even if the sign is off. In this experiment, it was not mentioned when and how PCMS was activated. The activation of the sign could be a challenging task. Furthermore, the study did not measure what effect the speed reduction had in relation to the volume and time of day. Despite the fact that the time periods, especially peak hours or weekday, can have clearly consequences in the result.

There are a number of messages that may be posted in dynamic message signs, DMS but which type can be the most useful and effective one. Some researchers suggested that an active DMS that display warning messages could directly affect driver performance because warning messages attracted the attention of the drivers. Borello and Ornitz grouped different types of messages given on a DMS in three different classes. Based on this classification, the authors measured what effect the message of sign had on driver performance with the measure of speed. In order to test the immediate influence of the DMS on driver performance, Borello and Ortniz defined a cone of influence of the DMS. The cone of influence was defined as a distance between the minimum and maximum line of sight for the sign to determine the best location of the speed detector for ideal readings. The cone was based on an angle of inclination of 7.5 degree in order that the driver may read the message in $918.0 \mathrm{ft}$ [9]. By carefully setting the cone parameters, it could be easily excluded drivers who could not see the message in the DMS from the analysis.

Rämä and Kulmala investigated the effectiveness of variable message signs warning of slippery road conditions and a minimum headway sign on driver behavior. Driver behavior was measured in terms of speed and headways variations under weather conditions, road surface and traffic conditions. The data was collected by using loop detectors at three test sites in Finland. To examine the effects of driver adaption and any possible novelty, an after study was established within two winters. Driver behavior was monitored at three measurement points, 536 - $1800 \mathrm{~m}$ upstream before the sign, 360 - $1100 \mathrm{~m}$ downstream after the sign and 7670 - 13,000 m downstream. The operators at the Traffic Management Centre TMC classified the road surface conditions in 
three categories, good, possibly slippery and verified slippery. The TMC operators switched on or off the sign to flashing mode depending on the presence of slipperiness. Results showed that the VMS decreased mean speed by $1-2 \mathrm{~km} / \mathrm{h}$ and increased the following distances. The positive effects were not found at all test sites. The effects were more significant before the sign than after the sign. However, at a distance 3 - $14 \mathrm{~km}$ after the signs, the mean speed increased slightly [10]. Rämä and Kulmala concluded that the variable message signs had other effects on driver behavior besides speed and headway.

Luoma, et al. designed a complementary study to investigate the other possible effect on reported driver behavior. In Luoma, et al. study, a combination of roadside and telephone interviews were done to drivers who encountered either of the signs that was used Rämä and Kulmala test sites. $2 \%$ of drivers who encountered the minimum headway sign declared that the sign had no effect on their behavior. Drivers reported other frequently effect of the slippery road conditions signs with a change in focus of attention, more concentration on their own driving, testing the road slipperiness and careful overtaking behavior. Many drivers informed that variable message signs improved their driving comfort. Luoma et $a l$. found that the slippery road condition sign had more effects during black ice than during snowfall conditions [11]. Therefore, the authors proposed dynamic weathercontrolled systems that can perform successfully in further study.

Rämä carried on another experiment on the Finish E18 test site to study the effect of weather-controlled variable speed limits and warning signs on driver behavior. The speed limits were automatically controlled by two road weather station in order to estimate the effects of road and weather conditions on speed. The road and weather conditions were classified as good, moderate or poor. These classifications were based on different parameters such as rain or snowfall, rain intensity, road surface, visibility and wind velocity. The main results from her study showed that the weather-controlled system decreased the mean speed and the standard deviation of speeds. The system had more effect on mean speed during summer season when the higher speed limits were allowed but weather conditions were not obvious, similar as winter conditions. Rämä reported that lowering the speed limit decreased the mean speed where the weathercontrolled system increased the homogeneity of driver behavior [12]. The experiments done by the author were carefully established and analyzed. She mentioned that there is a need for a more advanced system to recognize adverse road and weather conditions and low frictions.

The effect of VMS signs on drivers' behavior can vary with the ages of drivers. Older driver are more risky for crashes in work zones claimed Heaslip et al. in their research study. The authors studied the effectiveness of the work zones features implemented in Greenfield, Manssachusetts by collecting speed and video data over a fourmonth period. Video data was used to be able to approximate the age of the drivers and their maneuvers. The results showed that VMS had a positive effect for all drivers regardless that older drivers' speeds were obviously different than other drivers [13]. Heaslip et al. advised to develop advanced traffic analysis tools to get better understanding of driver behavior in work zone. They suggested the use of micro simulation and visualization techniques that should concern with new theory and structure by simulating expected driver behavior changes with other characteristics.

\section{Effect and Evaluation of Variable Speed Limits}

Variable speed limits VSL are variable messages signs that display speed limits. The displayed speed limits are determined by VSL algorithms that are based on real time traffic data. The VSL algorithms can have different design and provide different impact depending on the type and the control objective of the VSL system. Jiang et al. designed three types of algorithms for activating VSL on. These algorithms were based on high flow, queuing and weather conditions. For high flow conditions, the logic for determining new speed limit was retrieved from the speed-flow relationship curve, but for queuing conditions, the logic is done by reducing the upstream speed to a medium level speed level, between normal speed and the activated low speed limit. Jiang et $a l$. did not present the algorithm that is based on weather conditions. However, the authors demonstrated the effectiveness of VSL in high flow and queuing conditions by using a micro simulation model. The results from their model showed that VSL was able to achieve speed harmonization for motorway sections with low ramp and could contribute to the reduction of secondary crashes [14].

Nissan and Koutsopoulosb focused on the evaluation of the impact of advisory variable speed limit VSL on motorway capacity. A motorway control system was implemented E4 in Stockholm. The system was equipped with an Automatic Incident Detection to detect serious disturbances in the traffic streams. When the system noticed the disturbance, it generated automatically a suitable set of advisory speed limits for the approaching traffic [15]. The new displayed speed limit was based on a comparison to two speed threshold with low and high values. The values of these two speed threshold are not clearly identified in this study while these values are the main elements in triggering the VMS sign. However, the 
results from this study showed that advisory VSL did not give any important effect after its implementation. Nissan and Koutsopoulosb concluded that the obtained result was related to the advisory system used in Stockholm. The system should be obvious to motorists that the recommended speed limit was based on the serious disturbance detected where the system did not seem to be focus in this point. Besides, motorists ignored the advisory speed limit if it was not motivated by the traffic situation.

A two stage variable advisory speed limit system was developed and implemented by Kwon et al. The system was established for only three week period in February to March at one of the I-494 work zones in Twin Cities. The proposed system used real-time measurements at both downstream and upstream speed levels. It tried to reduce the speed of the upstream flow to reach the same level as that of the downstream traffic. Therefore, the advisory speed limit was determined from a function based on both upstream and downstream speed levels. Kwon et al. reached a promising result in reducing the speed differences by $25 \%$ to $35 \%$ along the work zone area during the 6:00 to 8:00 a.m. periods on weekdays. The estimation of driver compliance level by correlating the speed differences in both upstream and downstream was $20 \%$ to $60 \%$ [16]. In this research, the effects of the system were only checked for a short-time period. The effects of the signs should be measured on longer time period to study driver's compliance. Weather conditions play an essential role in the long term effectiveness of the advisory speed limit where the authors excluded this factor from their study.

Sandberg et al. investigated long term effectiveness of Dynamic Speed Monitoring Display, DSMD signs that were permanently installed in Minnesota, Washington and Dakota County. The study was conducted at speed reduction transition zones from a rural highway to urbanized area and was kept in one year duration. The overall results across all the test sites were clearly consistent in reductions in the 50th, 85th and 95th speeds averaging 6.3, 6.9 and $7.0 \mathrm{mph}$, respectively [17]. The authors mentioned that in order to draw conclusions for any long-term study the potential external influences other than the DSMS sign should be reviewed. However the mentioned review was not shown in their work. Simply they examined traffic speed and traffic volume with no check for the speed variation in relation with seasonal conditions. Sandberg et al. analysed the 24-hour average traffic speed and volume but vehicle speed could be extremely varied in rush hours than other time of the day. The variability of speeds during night time and daytime should also be analysed in their study.

McMurry et al. studied the effects of Variable Speed Limit VSL signs at work zones in Utah. VSL signs were tested against the existing static sign for about 3 months.
Night time standard deviation was analysed to examine the speed variation without construction interruption compared to daytime when work time was present. The results for this study showed that there were wide variations of average speed during the night time with regular static signs as compared to VSL signs [18]. Nevertheless the authors showed that night drivers and day drivers were more compliant to VSL signs than the regular static sign. However, the variability of speeds was only examined at short term but these variations might be very different at longer term. In this study, the speed limit posted in the sign at night time was $10 \mathrm{mph}$ higher than daytime meanwhile $10 \mathrm{mph}$ lower than the regular speed limit that usually posted on the road. The authors did not showed why the speed should be reduced by this amount and why the night posted speed in VSL could not be stepped back to the original speed limit. The effects of VSL on driver behaviour are summarized in Table 1.

\section{Effect of Vehicle Activated Sign}

Automatic speed warning signs, vehicle activated speed limit sign, or Active speed warning sign were considered as Vehicle Activated Sign, VAS. In fact, VAS has a system that is activated by driver's speed that exceeds a pre-set threshold. The sign had been developed and established in typical locations like dangerous entrances and curves, work zones and school zones. Different studies had been done in this area by presenting the effects of the use of VAS. Kathmann presented different approaches on how to assess the effectiveness of VAS. He pointed out that the approaches should be taken under two essential considerations that are important when assessing the effectiveness of VAS. The first consideration was that the observations must be done in a way that cannot be discovering by the driver. The second one was that the measurement for collecting and analysing the data should be cost-effective. The approaches could be either an inductive loop measurement or other empirical measurements. As empirical measurements, Kathmann proposed three methods such as car following method, video camera surveillance and voice recording. Car following method is used by a test vehicle with Datron sensor to get speed profile along the way instead of local speed data. Video camera surveillance and voice recording are used for analysing respective drivers braking behaviour and his familiarity with the road. Kathmann concluded that speed measurements should be collected before and after the installations of a VAS otherwise the assessment become difficult and time and work consuming. The best result reached from the methods was the car-following method [19]. Kathmann showed that speed reduction is not always based on the presence of VAS. Therefore there was a need to check the speed distribu- 
Table 1. Effect of VSL on driver behaviour.

\begin{tabular}{|c|c|c|c|}
\hline Authors & Year & Effect & Parameters used in the study \\
\hline Richard and Alex & 2010 & $\begin{array}{l}\text { Small beneficial reduction in mean speed and standard } \\
\text { deviation }\end{array}$ & Traffic volume and speed for some days \\
\hline Borello and Ornitz & 2010 & $\begin{array}{l}\text { Type of messages have different effect on mean speed, } \\
\text { standard deviation }\end{array}$ & Weekly average speed for each 20s \\
\hline Rämä and Kulmala & 2000 & $\begin{array}{l}1-2 \mathrm{~km} / \mathrm{h} \text { reduction in mean speed and increasing the } \\
\text { following distance in headways }\end{array}$ & $\begin{array}{l}\text { Speed, headways, road surface conditions in } \\
\text { three categories, good, possibly slippery and } \\
\text { verified slippery }\end{array}$ \\
\hline Rämä & 1999 & $\begin{array}{l}\text { More effect on mean speed and standard deviation in summer } \\
\text { season, increase the homogeneity of driver behavior }\end{array}$ & $\begin{array}{l}\text { Speed, road and weather conditions in three } \\
\text { categories good, moderate and poor }\end{array}$ \\
\hline Kwon et al. & 2007 & $25 \%$ to $35 \%$ speed reduction between 0600 and 0800 hrs & Speed, volume for every $30 \mathrm{~s}$ \\
\hline Sandberg et al. & 2008 & Average speed reduction by $7 \mathrm{mph}$ & Vehicle speed and volume \\
\hline Nissan and Koutsopoulosb & 2011 & No significant impact & Vehicle speed and flow density \\
\hline McMurry et al. & 2008 & Variations in speed was reduced in general & Vehicle speed at day and night time \\
\hline Jiang et al. & 2011 & Speed harmonization and better environmental impacts & High flow, queuing and weather \\
\hline
\end{tabular}

tion instead of average speed. He suggested integrating data from different VAS sources. That would give better knowledge for assessing the effectiveness of VAS.

Winnett and Wheeler established a full scale study of the effectiveness of over 60 signs installations of different types of vehicle-activated signs on rural single carriage way roads. This study had been conducted by TRL for the Department of Transport. They aimed to assess the effectiveness of the signs on both driver's speed and injury accident. Besides, they aimed to evaluate driver's understanding of the signs [20]. Vehicle Activated signs was depended on the trigger speed or pre-set threshold that switches on the sign. The speed threshold was set at the 50th percentile speed detected before the sign was installed. That was supposed to target half of drivers. In other previous studies, the speed thresholds were set at between the 75th and 81st percentile speeds. In that case, the signs targeted a very small population. However, when the threshold were set at between the 20th to 30th percentile speeds, thus targeted more drivers. The effect of the sign is clearly related to the trigger speed value in order that activating VAS. An early or late switch on could provide a negative or positive impact on the sign. So, what is the most suitable threshold trigger speed for a specific site and how can be determined? The activation threshold speeds should be dependent on different time periods under various conditions. To find out the right trigger speed that activates the sign, there is a need to analyse various trigger speed distribution in relation to average speed under diverse conditions.

In other study, Winnett et al. evaluated the effectiveness of an interactive fibre optic sign at a rural cross road. The main objective of this evaluation was in general to reduce speeds consistently and in particular target mo- torists at the top end of the speed distribution. The sign used was a warning sign with SLOW DOWN message, were switched on when driver's speed exceeded $46 \mathrm{mph}$. In previous studies in TRL, Winnett et al. suggested that it is possible to control the traffic speed by varying the threshold. The threshold established in their study was fixed to $46 \mathrm{mph}$. That was chosen to warn driver travelling above $50 \mathrm{mph}$ with $10 \%$ error margin on the detector used [21]. There was no basis why the threshold was fixed to $46 \mathrm{mph}$. VAS should only be activated when it should be activated, otherwise it could decrease its impact on the driver behaviour. In order to define the threshold of VAS, diverse potential factors should be checked.

In 2007, Mattox et al. conducted research study in South California DOT for development and evaluation of speed-activated sign by lowering speed in work zones. This study was based on a depth literature review of several speed reductions measures in work zones such as speed monitoring displays, changeable message signs with and without radar, vehicle activated signs. As a result of the assessment of these signs, it had been proven to provide remarkable effect in speed reduction, but due to their high cost, many transportation agencies did not established in all their work zones. The sign used in Mattox et al. research was vehicle activated sign, VAS which was the most cost effective sign among all other mentioned signs [22]. The predetermined speed threshold was set to the post speed limit with a $3 \mathrm{mph}$ buffer. Results from this research study showed a reduction in mean speed, 85 percentile speeds and percentage of vehicles exceeding the speed limit. This study was only evaluated short term effectiveness. However VAS might lose its effectiveness over time as drivers become habitu- 
ated to seeing them regularly.

The evaluation of effectiveness of the sign was established by studying before-after studies and concluded that the sign have an effect on reducing speeds, standard deviations or headways. The sign has its largest effect on drivers exceeding the speed limit excessively. All these evaluations were concerned when the sign is in operation but a few studies investigated the evaluation after removing the sign from the test site. Walter and Broughton observed the effect of speed indicator devices, SIDs after the sign is removed. SID is vehicle activated signs that display the real-time speeds of vehicle passing the device. In the study, Walter and Broughton installed ten SID at ten sites in South London in 2008 across all weekdays with different level of traffic flow and with periods of installation that are randomly assigned to weekdays. They designed the experiment to be balanced as far as possible by reducing the possible effect of external factors on the results. Across all sites, an overall speed reduction was $1.4 \mathrm{mph}$ [23]. The effect varied over time and differed across sites. SID showed a significantly effect on speeding drivers at all sites. However, there was no effect in speed after the sign was removed. Only a small reduction was lasting at those sites where the SID was in operation and had the most effect. Table 2 summarise the effect of VAS on driver behaviour and present the trigger speed value applied in the configuration of the sign.

\section{Summary and Conclusions}

In previous studies, authors pointed out that the effectiveness of VMS is related to the credibility of the message displayed on the sign. The displayed message should reflect road, traffic or weather conditions and VMS should only be triggered when such conditions deteriorate. The majority of drivers travel at a speed they consider reasonable, and safe for road, weather and environmental conditions. Therefore, unnecessary activation of the sign can provide negative effect of the sign on driver's behaviour. Additionally, the information shown in VMS were obtained after a long process in order to gather the traffic data, process it and compute an advisory message or a new speed limit. The time taken from this process could lead to late release of the information where it could lead to decreases in VMS reliability.

Weather conditions play an essential role in the effec- tiveness of VMS where most of the researchers excluded this factor from their study. There is a need for a more advanced system to recognize adverse road and weather conditions and low frictions. Therefore, dynamic weathercontrolled systems that can perform successfully in further study were proposed by some authors.

To assess the effectiveness of VMS or VAS, vehicle speed and traffic flow are the most used data in the previous studies. These data were basically analysed by averaging the speed or checking the standard deviation in relation to a specific time per days or days of week However, a reduction on the average speed is not always based on the presence of the sign. The sign should be placed at a site where it is not influenced by other factors such as junctions, roundabout or any traffic calming. That can lead to a misinterpretation of the results. The evaluation of the effectiveness of the VMS or VAS systems were conducted by using either empirical data from sensors such as loop detectors, camera or radar or by using micro simulation techniques. The empirical analyses were based on before-and-after VMS implementation on a specific test site. The results from this analysis were shown that VMS and VAS had an impact on driver behaviour in order on the effectiveness of both signs.

The relation between the design of VMS algorithm and the appropriate conditions are not clearin previous research studies [14]. Besides, for VAS, the trigger speed value was set with no basis. Authors did not consider the importance of the value of trigger speed on the impact of the sign on driver behaviour. The threshold value was set either $3 \mathrm{mph}$ or $5 \mathrm{mph}$ above the posted limit or $4 \mathrm{mph}$ under the posted speed limit. Which consequence could lead if the sign was trigger by all vehicle speed or vehicle speed that is above the posted speed limit or under the posted speed limit? All the experiments were established by providing a constant threshold value under different conditions with no consideration for a dynamic threshold value.

\section{Future Work}

Developing dynamic activation threshold value has not been thoroughly investigated in previous studies. The idea is to establish a model that attempt to predict the appropriate activation threshold value to the corresponding traffic and weather situations. Therefore, a systematic data collection will be focused in first place where traffic

Table 2. Effect of VAS on driver behavior.

\begin{tabular}{llll}
\hline Authors & Year & Effect & Trigger speed \\
\hline Mattox et al. & 2007 & Average speed reduction range $2.0-6.0$ mph & 3 mph over the posted speed limit \\
Winnett et al. & 1999 & Nearly $70 \%$ speed reductions for high end speeders. & 46 mph for 50 posted speed limit \\
Winnett and Wheeler & 2002 & Mean speed reductions between range $1.2-13.8$ mph & 5 mph above the posted speed limit \\
\hline
\end{tabular}


data will be collected but by varying the value of threshold activation value. The data is archived by various threshold values. Four databases shall be available for each threshold value: the database for daytime traffic; the database for night time traffic; the database for weekday's traffic and the database for weekends. Meanwhile, the traffic data shall further be integrated with weather data sources. Besides, data is analysed partly with the help of descriptive methods which may describe, organize and present the raw data and partly with the help of association rules which find common relationships between attributes that may be difficult to discover. Another aspect is to extract the speed distribution pattern of the road speed traffic data finding the similarities between vehicle speed and time. The similarities between different attributes can be obtained by using k-means clustering algorithms.

\section{REFERENCES}

[1] S. Nygårds and G. Helmers, "VMS: Variable Message Signs. A Literature Review," Swedish National Road and Transport Research Institute VTI Rapport 570A, Linköping, 2007.

[2] S. Nygårds, "Literature Review on Variable Message Sign 2006-2009," Swedish National Road and Transport Research Institute VTI Rapport 15A, Linköping, 2011.

[3] A. Nissan, "Evaluation of Variable Speed Limits: Empirical Evidence and Simulation Analysis of Stockholm's Motorway Control System,” Ph.D. Dissertation, Royal Institute of Technology, Stockholm, 2010.

[4] S. G. Charlton and P. Baas, "Assessment of Hazard Warning Signs Used on New Zealand Roads," Land Transport New Zealand Research Report 288, New Zealand, 2006.

[5] M. C. Taylor, A. Baruya and J. V. Kennedy, "The Relationship between Speed and Accidents on Rural SingleCarriageway Roads,” TRL Report, Wokingham, 2002.

[6] G. Nilsson, "Traffic Safety Dimensions and the Power Model to Describe the Effect of Speed on Safety," Lund Bulletin, 221, Lund Institute of Technology, Lund, 2004.

[7] T. Richard and D. B. Alex, "Effectiveness of Road Safety Messages on Variable Messages Signs,” Journal of Transportation Systems Engineering and Information Technology, Vol. 10, No. 3, 2010, pp. 18-23. doi:10.1016/S1570-6672(09)60040-4

[8] U. Firman, Y. Bai and Y. Li, "Determining the Effectiveness of Portable Changeable Message Signs in Work Zones," Proceedings of the 2009 Mid-Continent Transportation Research Symposium, Ames, 2009.

[9] C. Borello and S. E. Ortniz, "Dynamic Message Sign Influence on Driver Performance,” ITE 2010 Annual Meeting and Exhibit, Vancouver, 2010.

[10] P. Rämä and R. Kulmala, "Effects of Variable Message Signs for Slippery Road Conditions on Driving Speed and Headways," Transportation Research, Part F, Vol. 3, No. 2, 2000, pp. 85-94.

\section{doi:10.1016/S1369-8478(00)00018-8}

[11] J. Luoma, P. Rämä, M. Penttinen and V. Anttila, “Effects of Variable Message Signs for Slippery Road Conditions on Reported Driver Behavior,” Transportation Research Part F: Traffic Psychology and Behaviour, Vol. 3, No. 2, 2000, pp. 75-84. doi:10.1016/S1369-8478(00)00017-6.

[12] P. Rämä, "Effects of Weather-Controlled Variable Speed Limits and Warning Signs on Driver Behavior," Transportation Research Record, Vol. 1689, 1999, pp. 53-59.

[13] K. Heaslip, J. Collura and M. Knodler, "Evaluation of Work Zone Design Features to Aid Older Drivers,” 88th Annual Meeting of the Transportation Research Board, Washington DC, 11-15 January 2009.

[14] R. Jiang, E. Chung and J. Lee, "Variable Speed Limits: Conceptual Design for Queensland Practice," Proceedings of the Australasian Transport Research Forum, Adelaide, 28-30 September 2011.

[15] A. Nissan and H. N. Koutsopoulosb, "Evaluation of the Impact of Advisory Variable Speed Limits on Motorway Capacity and Level of Service,” Procedia Social and Behavioral Sciences, Vol. 16, 2011, pp. 100-109.

[16] E. Kwon, D. Brannan, K. Shouman, C.Isackson and B. Arseneau, "Development and Field Evaluation of Variable Speed Limit System for Work Zones,” Journal of the Transportation Research Board, No.2015, Transportation Research Board of the Natioanl Academies, Washington DC, 2007, pp. 12-18.

[17] W. Sandberg, T. Schoenecker, K. Sebastian and D. Soler, "Long-Term Effectiveness of Dynamic Speed Monitoring Displays (DSMD) for Speed Management at Speed Limit Transitions," 15th World Congress on Intelligent Transport Systems and ITS America's 2008 Annual Meeting, New York, 16-20 November 2008.

[18] T. McMurtry, M. Saito, M. Riffkin and S. Heath, "Variable Speed Limits Signs: Effects on Speed and Speed Variation in Work Zones," Transportation Research Circular: Maintenance Management, E-C135, 19-23 July 2009, pp. 159-174.

[19] T. Kathmann, "Assessment of the Effectiveness of Active Speed Warning Signs-Use of Inductive Loop Data or Empirical Data," 12th International Conference on Traffic Safety on Three Continents, Moscow, 19-21 September 2001.

[20] M. A. Winnett and A. H. Wheeler, "Vehicle-Activated Signs: A Large Scale Evaluation,” TRL Report TRL 548, TRL Limited, Crowthorne, 2002.

[21] M. A. Winnett, E. Woodgat and N. Mayhew, "Interactive Fibre Optic Signing at a Rural Crossroad (B1149 Felthorpe, Norfolk)," Transport Research Laboratory, TRL Report TRL 401, Crowthorne, 1999.

[22] J. Mattox, W. Sarasua, J. Ogle, R. Eckenrode and A. Dunning, "Development and Evaluation of a Speed-Activated Sign to Reduce Speeds in Work Zones," Transportation Research Record of the National Academies, Washington DC, 2007, pp. 3-11.

[23] L. Walter and J. Broughton, "Effectiveness of Speed Indicator Devices: An Observational Study in South London," Accident Analysis and Prevention, Vol. 43, No. 4, 2011, pp. 1355-1358. doi:10.1016/j.aap.2011.02.008 